\title{
Using Laser Scanners for Modeling and Analysis in Architecture, Engineering, and Construction
}

\author{
Daniel Huber, Member, IEEE, Burcu Akinci, Pingbo Tang, \\ Antonio Adan, Brian Okorn, and Xuehan Xiong
}

\begin{abstract}
Laser scanners are rapidly gaining acceptance as a tool for three dimensional (3D) modeling and analysis in the architecture, engineering, and construction (AEC) domain. Since 2001, our cross-disciplinary research team has been developing new methods for analyzing and modeling laser scanner data, with an emphasis on applications in the AEC domain. This paper provides an overview of our group's recent research efforts. Our work includes improving our understanding of the low-level aspects of laser scanner data, using comparison methods to analyze laser scanner data and derived models, and developing modeling and recognition algorithms to support the automatic creation of building models from laser scan data.
\end{abstract}

Index Terms-Building information models, laser scanning, reverse engineering.

\section{INTRODUCTION}

L ASER scanners are rapidly gaining acceptance as a tool for three dimensional (3D) modeling and analysis in the architecture, engineering, and construction (AEC) domain. Technological advances have led to laser scanners capable of acquiring range measurements at rates of tens to hundreds of thousands of points per second, at distances of up to a few hundred meters, and with uncertainties on the scale of millimeters to a few centimeters. Such sensors are well suited to densely capturing the geometry of building interiors and exteriors, process plants, and infrastructure. At the same time, software tools for processing and analyzing 3D point data sets (also known as point clouds) have been improving in their ability to handle the enormous point clouds produced by laser scanners and to integrate the use of point cloud data into CAD modeling software.

Since 2001, we have been working in a cross-disciplinary

D. Huber and Xuehan Xiong are with the Robotics Institute, Carnegie Mellon University; dhuber@cs.cmu.edu, xiong828@gmail.com.

Burcu Akinci is with the Civil and Environmental Engineering Department, Carnegie Mellon University; bakinci@ andrew.cmu.edu.

Pingbo Tang is with the Department of Civil and Environmental Engineering and Geodetic Sciences, Ohio State University; tangpingbo@ gmail.com.

Antonio Adan is with the Electrical Engineering Department, University of Castilla-La Mancha; Antonio.Adan@uclm.es.

Brian Okorn is in the Computer Engineering Department, Vanderbilt University; brian.e.okorn@vanderbilt.edu. research team to develop new methods for analyzing and modeling laser scanner data, with a focus on applications in the AEC domain. Our team consists of faculty and students from the Robotics Institute and the Civil and Environmental Engineering Department, as well as visiting faculty and students from other universities.

This paper provides an overview of our group's recent research on laser scanning for the AEC domain. The genesis of our research program was an NSF-funded project called ASDMCon (Advanced Sensor-based Defect Management at Construction sites) [1]. One key aspect of this project was the use of advanced sensors, such as laser scanners, to monitor for errors during the construction of a facility. In a series of case studies, we used laser scanners to scan construction sites periodically and created "as-built" models of each site (Figure 1). These as-built models were then compared to the facility's design model and geometric discrepancies were detected and flagged as potential defects.

In the course of the ASDMCon research, we learned some valuable lessons about using laser scanners for modeling buildings. First, it is important to understand the low-level limitations of laser scanners and the data processing workflows. Without such understanding, it can be difficult to tell the difference between a minor construction defect and noisy or uncertain data. Second, the concept of comparing what is measured (e.g., an as-built model) to what is expected (e.g., a design model) can be extended to many aspects of

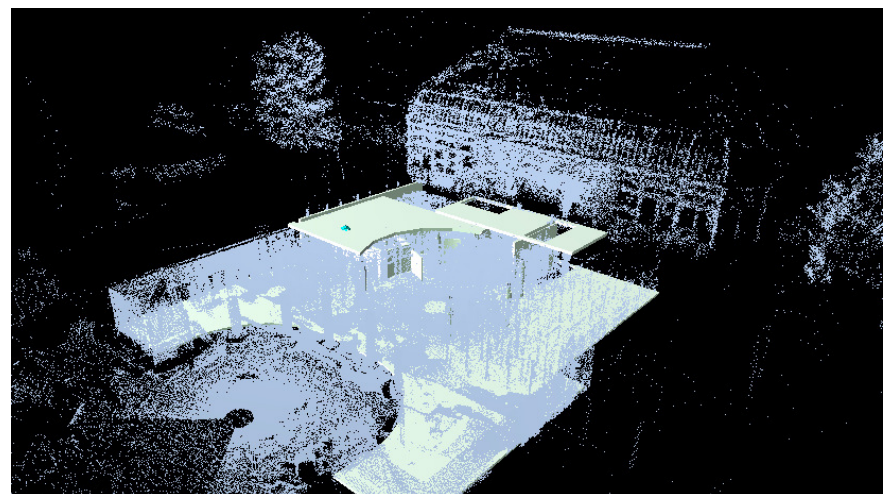

Figure 1. A case study from the ASDMCon project showing the design model overlaid on the as-built model. 
analysis in the AEC domain. Third, this comparison concept has limits, and there is a significant need to support facility modeling in situations where no design model is available. These lessons translated naturally into three distinct lines of research for our group: improving our understanding of the low-level aspects of laser scanner data; using comparison methods to analyze laser scanner data and derived models; and developing modeling and recognition algorithms to support the automatic or semi-automatic creation of building models from laser scan data. The next three sections describe our work on these topics in more detail.

\section{UNDERSTANDING THE LIMITATIONS OF LASER SCANNERS}

Reading the product literature for commercial laser scanners, one would conclude that these devices work perfectly for sensing all types of surfaces that you might want to scan. In reality, laser scanners have difficulty with many types of surfaces that occur commonly in the built environment, including low-reflectance surfaces (e.g., anything painted black), specular surfaces (e.g., shiny metal and mirrors), and transparent or translucent surfaces (e.g., windows).

Artifacts can even occur on benign surfaces that are considered easy cases. For example, in nearly every laser scan, phantom measurements that don't correspond to any physical surface appear at depth boundaries. These artifacts are known as mixed pixels because they are caused by the laser spot straddling two surfaces that lie at distinctly different distances from the sensor. Mixed pixels can cause an algorithm to hallucinate structures that don't exist, and they can cause significant error in the dimensions of modeled surfaces. We have conducted two research studies to better understand these effects.

\section{A. Mixed Pixel Detection}

Since all laser scans contain mixed pixels, virtually all laser scanner software includes some filtering algorithm to remove this invalid data. A number of relatively simple algorithms have been proposed - such as triangulating the point cloud and removing long edges. A few more complex algorithms have been put forth as well.

Many people consider mixed pixel detection to be a solved problem. We were interested in determining just how "solved" this problem really is. We performed a comparative analysis of the performance of the mixed pixel detection algorithms that we identified in the $3 \mathrm{D}$ vision literature and those algorithms that scanner software manufacturers were willing to disclose [6]. We scanned several different types of environments - a highway bridge, a building exterior, a research lab, and a public building interior. In each environment, we manually labeled the mixed pixels in several patches and then evaluated the performance of each algorithm on the labeled data (Figure 2). What we found was somewhat surprising. Although some algorithms performed better than

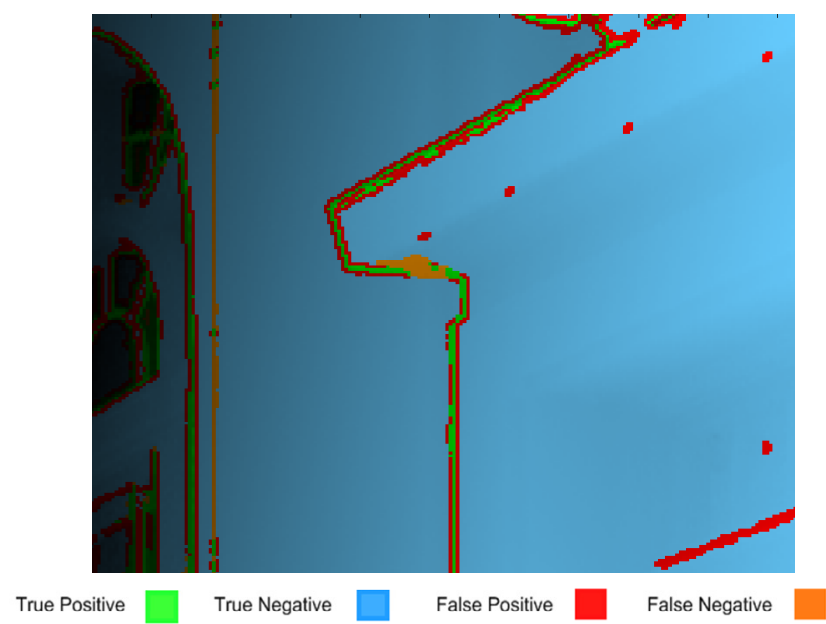

Figure 2. An example of the best performing mixed pixel detection algorithm applied to a decorative window overhang on a building exterior. Notice that the algorithm generates a significant number of false positives and also some false negatives on the same boundary.

others, none of the algorithms performed all that well. In fact, the best algorithm could only detect $92 \%$ of mixed pixels with a $7 \%$ false alarm rate. Practically, this means that in order to remove most of the mixed pixels, a significant number of valid pixels must be removed, too, and even still, some mixed pixels must be manually removed. We conclude that the mixed pixel problem is not quite as solved as we originally thought.

\section{B. Modeling Edge Loss at Depth Boundaries}

In the AEC domain, it is common practice to assess geometric properties of structures, such as the width of a beam or the length of a wall. Laser scanners can replace traditional physical measurement methods by enabling noncontact virtual measurements within the laser scan data. Unfortunately, simply making measurements in the laser scan data can lead to significant error when the measurement involves a depth boundary. The misestimation is caused partly by the eroding effect of mixed pixels and partly by the fact that laser scanners sample the environment at discrete intervals. In one example, we observed that a $91 \mathrm{~cm}$ bridge column lost $5.6 \mathrm{~cm}$ of its width.

Based on these observations, we undertook a study to develop a model to predict the amount of edge loss that will occur at a depth boundary as a function of the various factors that could influence the effect [5]. These factors include the laser spot size, angular resolution, range, angle of incidence, and scanner rotational velocity. One motivation for developing this model is that if we can predict the amount of edge loss that will occur in a given situation, we can use that prediction to compensate for the actual edge loss and, therefore, improve the accuracy of measurements.

We validated our edge loss model using controlled experiments in which we scanned a target with known widths 

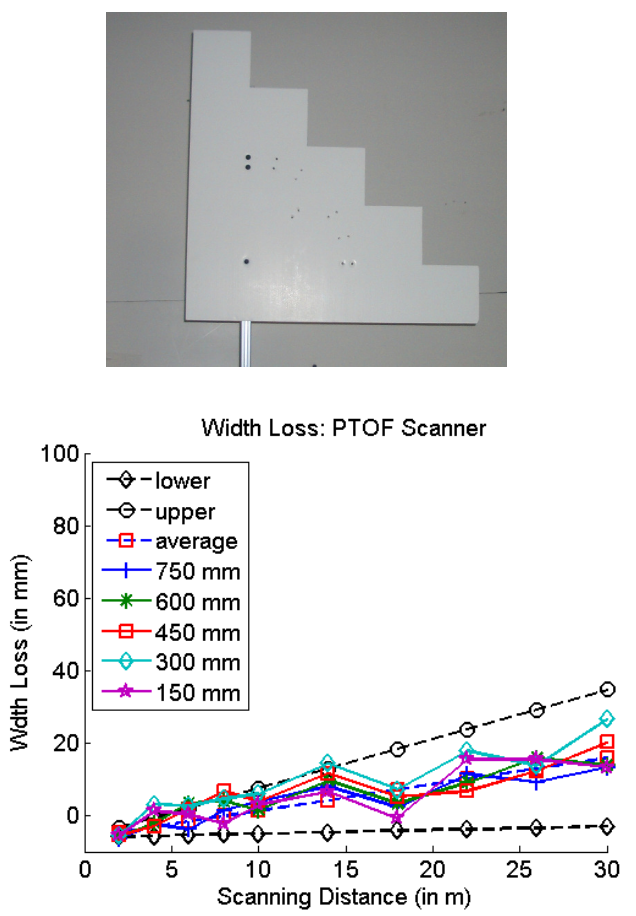

Figure 3. Modeling edge loss. Top: The test target used for controlled experiments to validate the edge loss model. Bottom: A plot showing the predicted edge loss for a specific pulsed time of flight (PTOF) laser scanner as a function of scanning distance. The first three lines in the legend are the predicted upper bound, lower bound, and average edge loss. The remaining lines are the measured edge loss for different widths in the test target.

and heights and also measured the corresponding widths and heights in the resulting point cloud data (Figure 3). We then compared the edge loss in the virtual measurements to the amounts predicted by our model. The measured results closely matched the model predictions across a wide range of testing conditions and even across scanners using different underlying technologies. Interestingly, our model predicted (rather counter-intuitively) that in some situations, the boundaries could actually grow rather than erode. Our experiments confirmed that this effect does indeed occur in practice. We also validated the method using field experiments on infrastructure inspection tasks. Using the edge loss compensation from our model prediction, we were able to reduce the error for measurements by an average of $80 \%$ for one scanner, for example, reducing a $5 \mathrm{~cm}$ error to less than $1 \mathrm{~cm}$.

\section{USING LASER SCANNERS FOR ANALYSIS}

Despite the various artifacts that laser scan data can contain, there are many applications where the data can be used to effectively analyze surfaces. Our projects in this line of research include using laser scanners to inspect concrete surfaces for flatness and detecting deviations between laser scan data and building models derived from the data.

\section{A. Analyzing Surface Flatness}

In many construction projects, it is important to ensure that concrete surfaces are flat to within a specified tolerance or that they conform to some designed surface shape. Traditionally, flatness assessment is performed manually, either with a long straightedge or with an elevation measuring device, such as a rolling profiler. These methods are relatively time-consuming and only make sparse measurements. Laser scanners, in contrast, can be very fast and can make dense measurements.

It is not clear whether laser scanners can accurately measure surface flatness with sufficient accuracy, because the required tolerances are often smaller than the uncertainty of a scanner's range measurements. One strategy to improve the accuracy of noisy data is to aggregate information spatially, trading off spatial resolution in the process.

Given the relatively high noise levels of laser scanner measurements compared to the flatness tolerance, we asked the question, "How large does a deviation from perfectly flat need to be before a laser scanner can detect it?" To answer this question, we developed a framework for controlled and objective evaluation of flatness defect detection algorithms [4]. The framework consists of a test bed populated with flatness defects of various diameters and thicknesses, measures for objectively evaluating algorithm performance, and a testing procedure for scanning the test bed, applying a flatness detection algorithm, and reporting the performance results (Figure 4). We also developed several flatness defect detection algorithms and compared their performance using
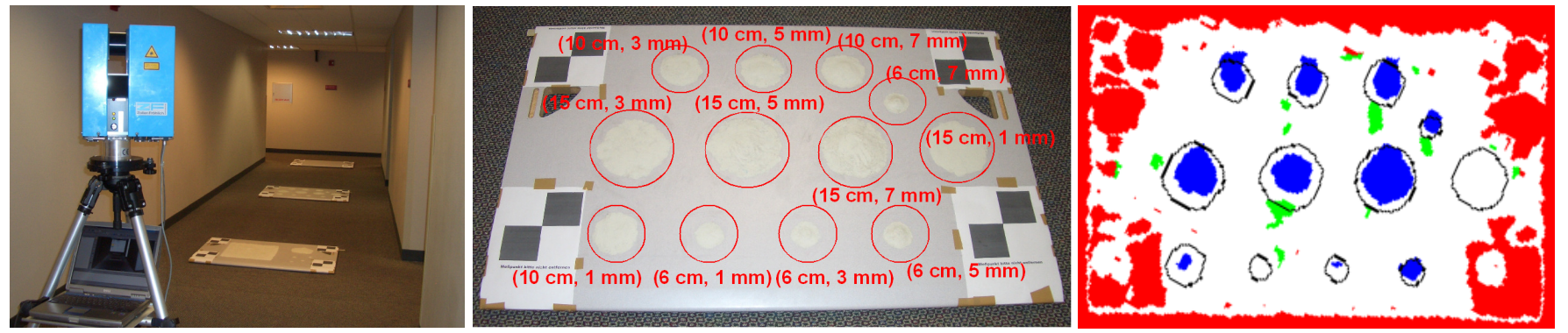

Figure 4. Analysis of surface flatness. Left: An overview of our experimental setup. Center: Our flatness defect test bed with circular defects of varying diameters and thicknesses. Right: A map showing regions of correct detection (blue), false detections (green), and detections in masked border regions (red). 
this framework and data from multiple laser scanners. The results of our experiments show that it is possible to detect surface flatness defects as small as $3 \mathrm{~cm}$ across and $1 \mathrm{~mm}$ thick from a distance of $20 \mathrm{~m}$.

\section{B. Quality Assurance for As-built building models}

Analysis of laser scan data can also play a central role in verifying the accuracy of as-built building models. Typically, when a company or organization needs a model of a building, they will contract the work out to a laser scanning service provider. The resulting model must be verified for accuracy through a quality assurance (QA) process. One approach for QA of as-built models is to acquire physical measurements from the building and compare them with virtual measurements of corresponding structures in the model. This approach has several disadvantages, chief among them are requirement of physical access to the facility, sparse coverage of potential locations of errors, lack of intuition into the source of detected errors, and the time-consuming nature of the process.

Borrowing from the idea of comparing the design model to the as-built model that we used in the ASDMCon project, we are developing an alternative approach. Here, we compare the as-built model to the raw point cloud data. If the as-built model faithfully represents the point cloud data, differences in the data sets should be minimal. Any regions of the as-built model having large deviations from the point cloud data are potentially problematic. Commercial reverse engineering software already supports this type of analysis, but it is not yet commonly used for QA in the AEC community.

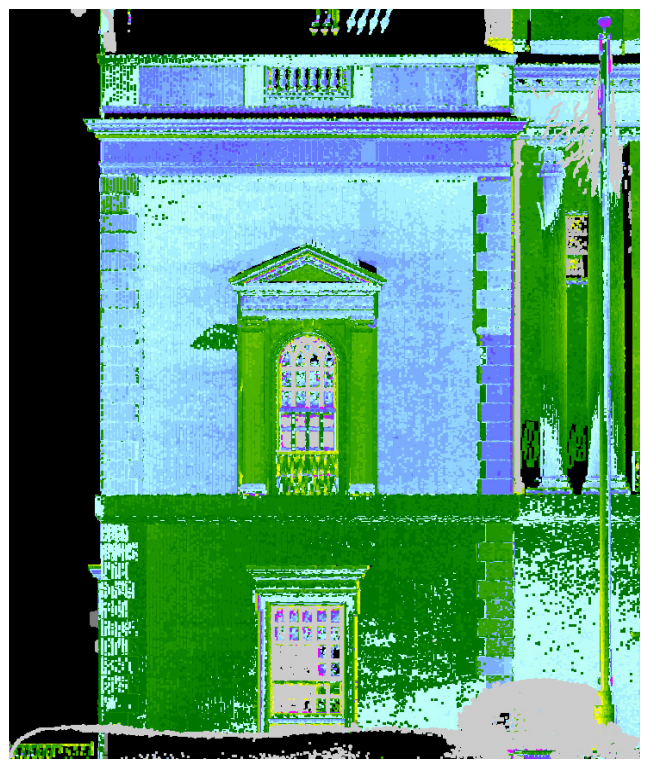

Figure 5. An example of modeling errors detected by the deviation analysis method for QA. The wall of the upper floor is offset from the wall of the lower floor by approximately $3 \mathrm{~cm}$, but it was modeled using two walls on the same plane. The color coding indicated the amount of difference between the point cloud and the model, with blue being $3 \mathrm{~cm}$ and green being under $0.5 \mathrm{~cm}$.

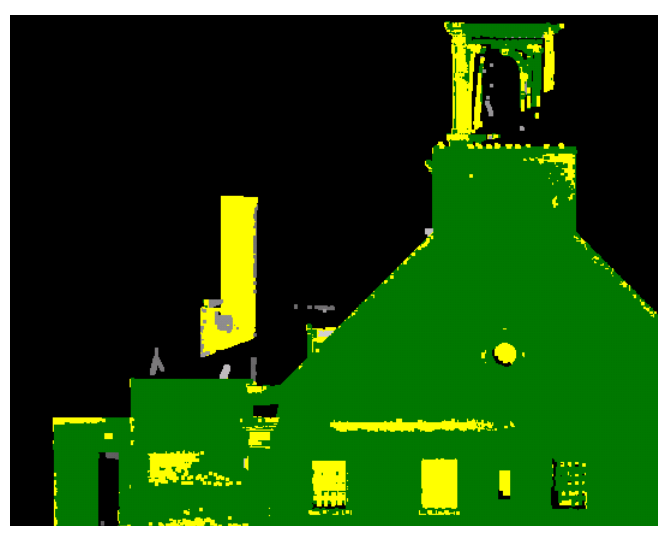

Figure 6. Another modeling error example. In this case, the chimney model is offset from the point cloud. The width of the chimney was modeled $10 \mathrm{~cm}$ larger than the width in the point cloud. Here, the deviation are thresholded, so that any deviation over $2.5 \mathrm{~cm}$ is shown in yellow, and deviations below the threshold are shown in green.

Working with the U.S. General Services Administration (GSA), we have conducted a number of case studies to evaluate this deviation analysis concept. Our results indicate that modeling errors are more common than one would expect. The exterior façades of a three storey building analyzed in one case study resulted in a significant number of errors that were beyond the accuracy tolerance set by the GSA (Figure 5 and Figure 6).

We are currently working on conducting more extensive case studies and assisting the GSA in integrating the process into their workflow. Our current approach utilizes commercial software for the analysis, but the process could benefit from automation. For example, windows often show up as significant errors, but closer inspection often reveals that the point cloud corresponds to unmodeled objects, such as curtains inside windows. Automated methods to detect the windows and analyze them using different criteria could reduce the number of false alarms significantly.

\section{USING LASER SCANNERS FOR MODELING}

Our QA analysis work shows that the process of creating building models from point clouds can be error-prone. The process is also very labor intensive. Each component in the model must be individually created and fit to the data. Frequently, the model needs to be augmented with semantic information, such as the identity of walls, windows, doorways, etc. These semantically rich models are known as building information models (BIMs).

The automatic creation of BIMs from laser scan data is considered to be a "grand challenge" type of problem by the AEC community (though it does not have an official designation as such). One of the primary reasons that as-built BIMs have not yet gained wide acceptance in the community is the difficulty and expense associated with their creation, and automating the process would likely lead to a paradigm 


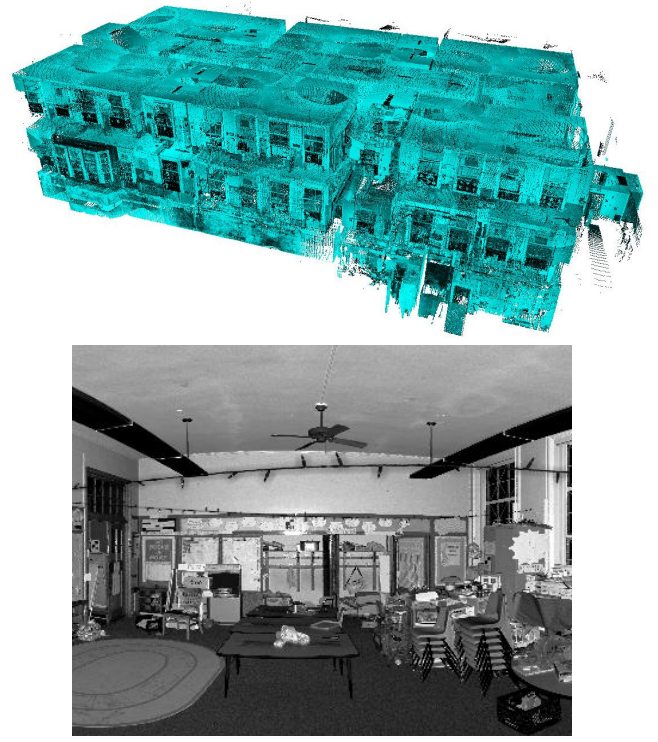

Figure 7. A schoolhouse which we are using as a case study for modeling. Top: The combined point cloud from all of the scans. Bottom: A portion of the reflectance image from one scan, showing the degree of clutter in the natural environment.

shift in the way that facilities are designed and maintained throughout their lifecycle.

For the past year, the focus of our modeling research has been on solving the problem of automated creation of as-built BIMs. We have already made some progress toward achieving our goal. Ultimately, we envision a robotic system that could navigate through a facility, obtaining 3D measurements, and then would automatically produce a highly-accurate, semantically rich building model. In the robotics community, many researchers have studied the problem of mapping indoor facilities. The main distinction between our work and prior research is that robotics applications are typically more concerned with supporting robotic navigation or generating photo-realistic models, whereas the primary focus of as-built BIMs is on completeness and accuracy. In our initial work, we are concentrating on the components that comprise the building envelope - walls, ceilings, floors, windows, and doors/doorways. We are interested in modeling buildings that are in active use, which means that the environments will contain significant clutter and occlusions from furniture, fixtures, pictures on the walls, and so on (Figure 7).

\section{A. Floor Plan Modeling}

One reason to use laser scanners in a facility is to create blueprints of the as-built condition, which may differ from the design blueprints (assuming they still exist). We are working on methods to automatically create accurate floor plan models of building interiors using laser scan data. Our approach is based on the observation that when the 3D points are projected onto the ground plane, the projected point density is frequently highest at the wall locations, since those surfaces are generally vertically oriented (Figure 8). Using this two dimensional (2D) projection, linear structures can be extracted using image processing operations like the Hough transform. We also observed that the amount of clutter in an environment varies as a function of the height above the floor. By strategically selecting cross sections with minimal clutter, we can significantly improve the chances that a given wall surface will be observed without being occluded by clutter. Finally, we are developing measures to objectively evaluate the performance of floor plan modeling algorithms, since, to our knowledge, no such methodology has been established.

One of the limitations of our approach is that it does not model windows and doorways - they are just regions where no linear structures were detected. The results could be further improved if we explicitly detect and model these types of components and if we incorporate full 3D reasoning into the process - extensions that we are currently investigating.

\section{B. Context-based Recognition of Building Components}

The structures which we are interested in modeling (walls, ceilings, and floors) are often planar surfaces, so it is sensible to try modeling planar regions initially, even if some surfaces require more complex geometries. Our goal is not only to model the surfaces, but also to identify the surface types and to establish connectivity relationships between them.

Many researchers have modeled building interiors as a collection of planar surfaces. In some cases, the planar surfaces are classified into categories like wall, ceiling, and floor. The predominant method used in previous research has been to encode the classification rules in a semantic network $[2,3]$. The downside to such an approach is that the rules must be manually encoded and would likely be brittle when faced with novel environments. In contrast, we use a learning-based approach, which can adapt to new data when it is encountered.
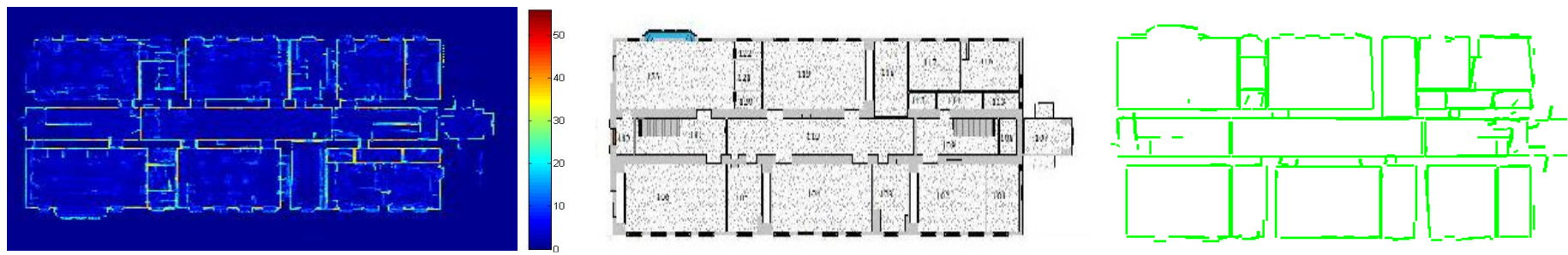

Figure 8. An example of automatic floor plan modeling. Left: The histogram of density of points projected onto the ground plane for one storey of the schoolhouse data shown in Figure 7. Center: The ground truth location of the walls (taken from a manually created BIM). Right: The floor plan model produced by our approach. 

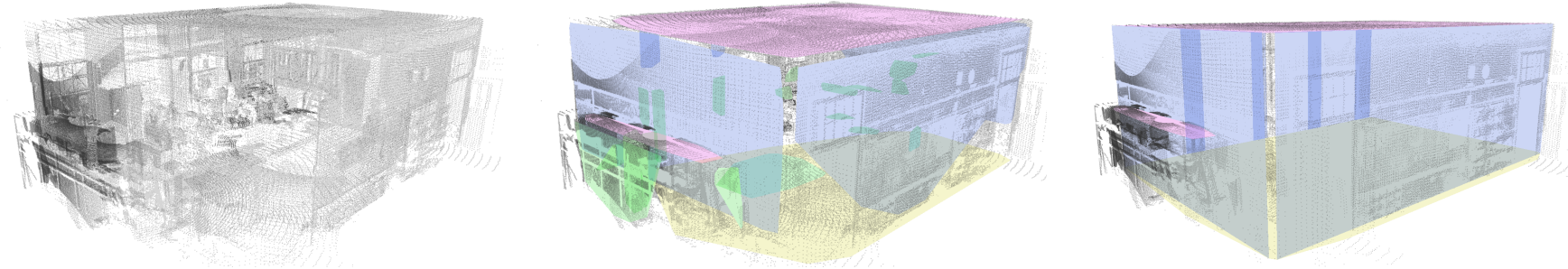

Figure 9. Context-based recognition of building components. Left: The raw point cloud for a room. Center: Automatically labeled planar regions $($ blue $=$ wall, red $=$ ceiling, yellow $=$ floor, green $=$ clutter). Right: The recognized components with the clutter removed and boundaries cleaned up.

We are investigating methods that leverage context for the recognition process. In some cases, it can be difficult to distinguish similar-looking components based on the components alone. A window looks much like a doorway, and a table has similar geometry to a floor. We believe that such ambiguous objects can be more easily recognized by considering the context of the objects. For example, a doorway is more likely to be located near the floor, and a floor is usually adjacent to a wall at the bottom, while a table is not. These types of contextual relationships can be represented and reasoned about using machine learning techniques. Our initial results indicate that the methods give good results for recognizing walls, floors, and ceilings, and we are beginning to look at how windows, doors, and doorways can be incorporated into the framework (Figure 9).

\section{Detailed Wall Modeling}

Although it is possible to model walls as planar patches, they are actually much more complex entities. Walls can be interspersed with windows and doorways, parts may be occluded by furniture and other objects, and items like bookshelves, clocks, and pictures may be attached or adjacent to the walls. We are investigating methods to more accurately model the detailed aspects of walls by explicitly reasoning about occlusions and their impact on the wall model (Figure 10). As part of this research, we are also developing methods to automatically recognize and model windows and doorways, even when they are partially occluded. When integrated with our context based recognition framework, these detailed wall modeling algorithms will enable us to model all of the building components that we are targeting in our automatic as-built BIM modeling algorithms.

\section{CONCLUSION}

Our research on using laser scanners in the AEC domain is ongoing in each of the three lines of inquiry that we have described. We are particularly encouraged by the progress we have made so far on the automatic creation of as-built BIMs. However, we recognize that much remains to be done. There are numerous open research issues in all of these areas, and exciting recent developments in 3D sensors, such as improvements in flash LIDAR technology, have the additional potential of opening up entirely new areas for exploration.

\section{REFERENCES}

[1] B. Akinci, F. Boukamp, C. Gordon, D. Huber, C. Lyons, and K. Park, "A Formalism for Utilization of Sensor Systems and Integrated Project Models for Active Construction Quality Control," Automation in Construction, vol. 15, no. 2, pp. 124-138, February 2006.

[2] H. Cantzler, R. B. Fisher, and M. Devy, "Improving architectural 3D reconstruction by plane and edge constraining," in Proceedings of the British Machine Vision Conference, 2002, pp. 663-72.

[3] A. Nüchter and J. Hertzberg, "Towards Semantic Maps for Mobile Robots," Journal of Robotics and Autonomous Systems (RAS), vol. 56, no. 11, pp. 915-926, November 2008.

[4] P. Tang, B. Akinci, and D. Huber, "Characterization of Three Algorithms for Detecting Surface Flatness Defects from Dense Point Clouds," in Proceedings of the IS\&T/SPIE Conference on Electronic Imaging, Science and Technology, San Jose, CA, January, 2009.

[5] P. Tang, B. Akinci, and D. Huber, "Quantification of Edge Loss of Laser Scanned Data at Spatial Discontinuities," Automation in Construction, vol. 18, no. 8, pp. 1070-1083, December 2009.

[6] P. Tang, D. Huber, and B. Akinci, "A Comparative Analysis of Depth-Discontinuity and Mixed-Pixel Detection Algorithms," in Proceedings of the International Conference on 3-D Digital Imaging and Modeling (3DIM), August, 2007, pp. 29-38.
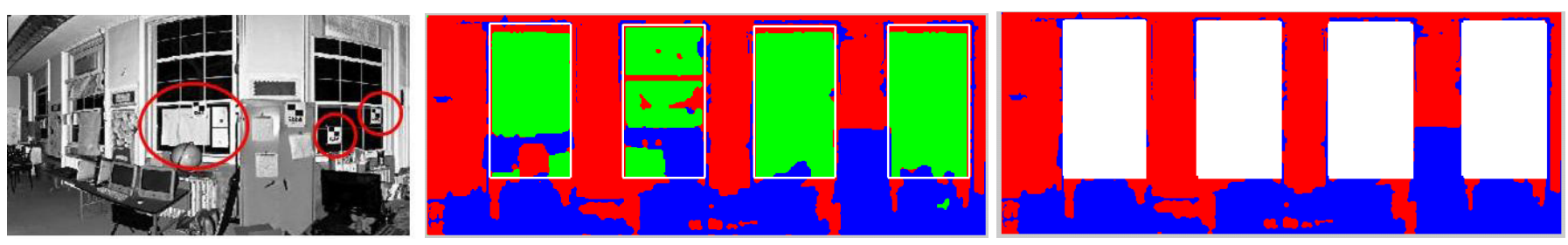

Figure 10. Detailed wall modeling. Left: A reflectance image showing the high amount of wall occlusion and objects blocking regions of the window. Center: Wall regions are classified based on occlusion reasoning from all sensor viewpoints $(\mathrm{red}=$ observed surface, green $=$ free space, blue $=$ occluded). Right: Final wall model after window detection and localization. 